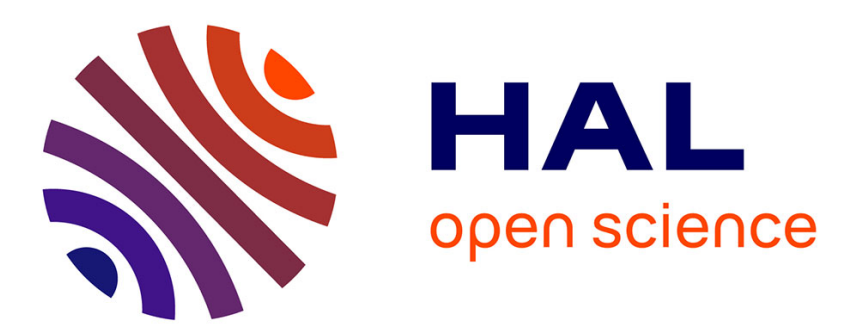

\title{
De l'interpolation optimale au 4D-Var: l'émergence d'un nouveau dominant design en assimilation de données météorologiques
}

Sylvain Lenfle

\section{- To cite this version:}

Sylvain Lenfle. De l'interpolation optimale au 4D-Var: l'émergence d'un nouveau dominant design en assimilation de données météorologiques. La Météorologie, 2018, Spécial Anniversaire 25 ans, 100, pp.37-46. 10.4267/2042/65141 . hal-03161142

HAL Id: hal-03161142

https://hal-cnam.archives-ouvertes.fr/hal-03161142

Submitted on 5 Mar 2021

HAL is a multi-disciplinary open access archive for the deposit and dissemination of scientific research documents, whether they are published or not. The documents may come from teaching and research institutions in France or abroad, or from public or private research centers.
L'archive ouverte pluridisciplinaire HAL, est destinée au dépôt et à la diffusion de documents scientifiques de niveau recherche, publiés ou non, émanant des établissements d'enseignement et de recherche français ou étrangers, des laboratoires publics ou privés. 
Rubrique : Histoire

\title{
De l'interpolation optimale au 4D-Var : l'émergence d'un nouveau dominant design en assimilation de données météorologiques
}

\author{
Sylvain Lenfle \\ Conservatoire National des Arts et Métiers (LIRSA), Paris et i3-CRG (UMR 9217), \\ École Polytechnique, Palaiseau. Mail : sylvain.lenfle@lecnam.net
}

Article accepté pour publication dans le numéro 100 de la revue La Météorologie (CSMF, Météo-Climat), février 2018. La publication originale sera disponible sur le site: http://irevues.inist.fr/la-meteorologie

\begin{abstract}
Résumé
Au cours des années 1990, les méthodes d'assimilation de données utilisées en prévision numérique du temps ont connu un changement radical. Les méthodes dites d'interpolation optimale, qui s'étaient imposées dans les années 1980, ont laissé place aux méthodes dites variationnelles, qui ont notamment permis de mieux exploiter les données satellitaires. Cet article analyse les origines et le déroulement de ce basculement à partir d'un travail d'histoire des sciences centré sur la question de l'assimilation des luminances mesurées par les sondeurs atmosphériques. Il montre comment les limitations observées de l'interpolation optimale aboutissent au milieu des années 1980 à une rupture conceptuelle, l'assimilation variationnelle, puis au lancement du projet IFS/Arpege qui va permettre la mise en œuvre opérationnelle de la méthode au Centre européen pour les prévisions météorologiques à moyen terme (CEPMMT) et à Météo-France dans la seconde moitié des années 1990. Ceci aboutira, après 10 années de travail acharné, à une amélioration significative de la qualité des prévisions.
\end{abstract}

From optimal interpolation to 4D-Var: the emergence of a new dominant design in meteorological data assimilation

\begin{abstract}
During the 1990s, data assimilation methods used in numerical weather prediction have undergone a radical change. Optimal interpolation techniques, which have become dominant in the 1980s, have been replaced by the variational approach. These new methods allow in particular a better use of satellite data. This article analyzes the origins and unfolding of this changein from a history of science standpoint centered on the assimilation of radiances measured by atmospheric sounders. We show how the limitations of optimal interpolation lead in the mid-1980s to a conceptual breakthrough, variational assimilation, and to the subsequent launch of the IFS/ARPEGE project leading to the implementation of the method by the European Centre for Medium-Range Weather Forecasts (ECMWF) and Météo-France in the second half of the 1990s. This has led, after ten years of hard work, to a substantial increase in the accuracy of weather forecasts.
\end{abstract}


L'objectif de cet article est d'analyser le changement majeur intervenu pendant les années 1990 dans les méthodes d'assimilation de données en prévision numérique du temps (PNT). Le but de ces algorithmes mathématiques est de générer les conditions initiales utilisées dans les modèles qui soient les meilleures possibles afin d'améliore la qualité de la prévision. En effet, en une décennie, les principaux centres mondiaux de PNT ont basculé des méthodes dites d'interpolation optimale aux méthodes d'assimilation dites variationnelles (3D ou 4D-Var). Cette histoire a déjà fait l'objet de différentes publications dans La Météorologie (en particulier Pailleux, 1996 ; Rabier et al., 2000 ; Pailleux et al.,, 2015 ; Mahfouf et al., 2017) qui ont permis d'éclairer et de documenter différents aspects de ce processus. Notre approche est quelque peu différente. D'abord car nous ne sommes pas météorologue de formation mais enseignantchercheur en management de l'innovation. Nous abordons donc le sujet avec les concepts de notre discipline qui, nous le pensons, permettent d'éclairer d'un jour neuf cette transition technologique en particulier celui, que nous présenterons, de dominant design qui permet de comprendre la complexité du processus. Ensuite ce travail s'inscrit dans une recherche en cours avec le Centre national d'études spatiales (Cnes) sur les processus d'innovation dans le domaine de l'observation de la terre. La question de l'assimilation des luminances énergétiques ${ }^{1}$ mesurées par les sondeurs atmosphériques embarqués sur les satellites météorologiques nous servira donc de fil conducteur. Le cas nous a semblé particulièrement intéressant car il s'écoule 20 ans entre le lancement du premier sondeur atmosphérique opérationnel (HIRS-2 en 1978) et la démonstration d'un impact positif de ces données pour l'amélioration des prévisions sur l'hémisphère nord (Andersson et al., 1994 ; Derber et Wu, 1998)². Analyser les causes de cette durée nous a conduit à entreprendre un travail d'histoire des sciences basé à la fois sur la littérature (en histoire des sciences et en météorologie) et sur des entretiens avec les acteurs de cette transition (à savoir dans l'ordre Jean Pailleux, Olivier Talagrand, Philippe Courtier, François-Xavier Le Dimet, Jean-Noël Thépaut et Florence Rabier). Le cas des luminances nous est alors apparu comme symptomatique des difficultés à innover, c'est-à-dire à utiliser de nouvelles données dans un système opérationnel. Nous présenterons dans une première partie les difficultés pour faire passer une mission satellitaire du domaine de la recherche à celui de l'opérationnel. Après avoir introduit le concept de « dominant design », nous expliquerons les raisons qui expliquait les difficultés d'assimilation de certaines données dans les modèles de PNT avec les premières méthodes d'assimilation (dites d'interpolation optimale). Nous montrerons ensuite comment une nouvelle méthode d'assimilation (dite variationnelle) est apparue et comment elle a permis d'utiliser de manière plus efficace ces données satellitaires. Finalement, les différentes étapes de la transition vers ce nouveau dominant design seront illustrées à travers le projet collaboratif IFS/Arpege entre le Centre européen pour les prévisions météorologiques à moyen terme (CEPMMT) et Météo-France.

\footnotetext{
${ }^{1}$ La luminance énergétique est une grandeur radiométrique qui mesure le flux d'énergie dans une certaine direction et dans une certaine gamme de longueur d'onde du spectre électromagnétique. Comme nous allons le voir, elle est utilisée en PNT pour mesurer des paramètres atmosphériques indispensables à la PNT, en particulier la température et l'humidité de l'atmosphère.

${ }^{2}$ On peut allonger ce délai à 30 ans si l'on considère qu'une dizaines d'années ont été nécessaires pour la phase exploratoire. Le premier sondeur expérimental SIRS-A a en effet été lancé en 1969. HIRS-2 dérive quant à lui du sondeur expérimental HIRS-1 lancé en 1975. Une dizaine d'années ont été nécessaires pour disposer des données fiables selon les critères météorologiques (1978 selon Courtier, 1997).
} 


\section{Franchir les « vallées de la mort » jusqu'à l'utilisation opérationnelle}

Le cas est d'autant plus intéressant que les difficultés à utiliser de nouvelles données dans les systèmes opérationnels d'assimilation pour la prévision numérique du temps (PNT) ne sont qu'une illustration du problème générique de l'utilisation des données spatiales, problème qui dépasse la seule météorologie. Ainsi, dans un rapport de 2003 intitulé Accelerating the transition from research to operations, le National Research Council (NRC) américain identifie en effet deux vallées de la mort que doivent franchir les données spatiales (figure 1).

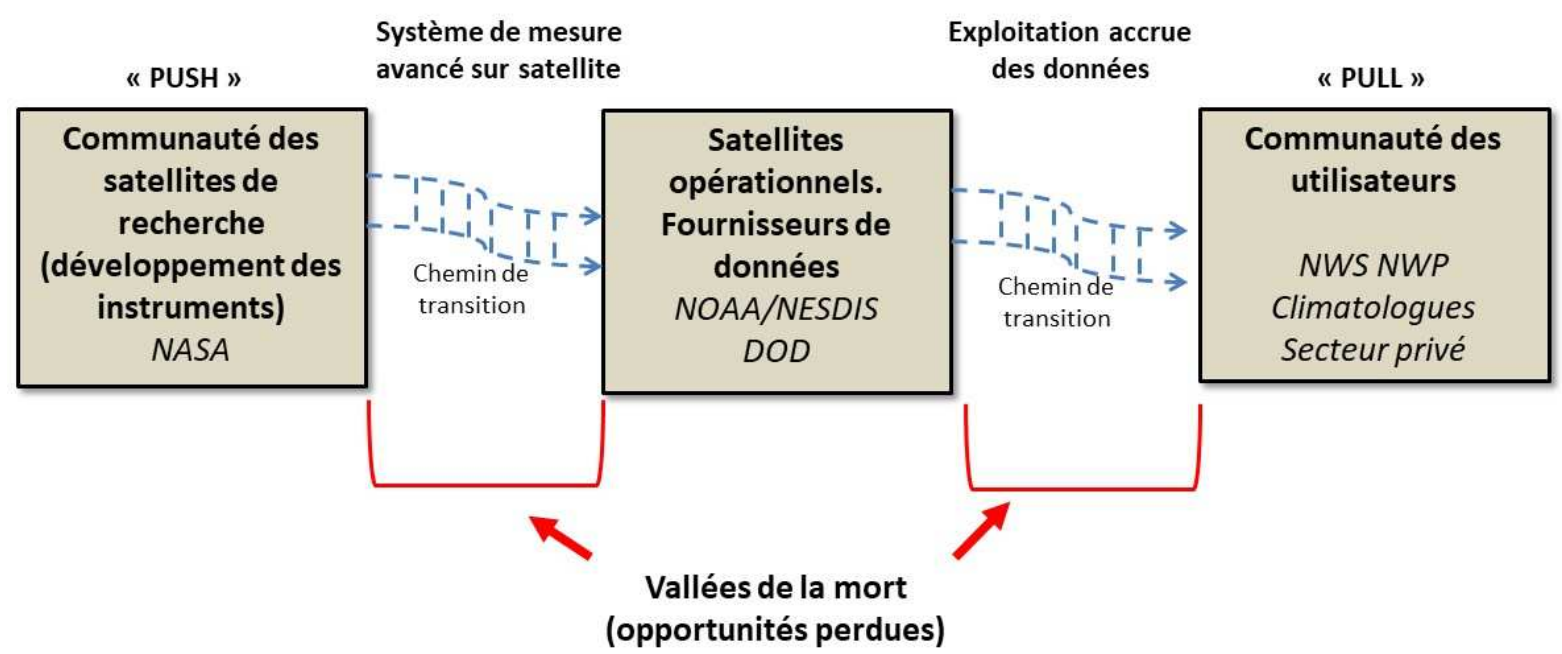

Figure 1. Explication des vallées de la mort dans le développement et l'utilisation des données satellitaires (d'après laNRC en 2003).

Dans un scénario idéal, un satellite de recherche donne naissance à une série de satellites opérationnels produisant des données qui sont ensuite intégrées par les utilisateurs pour fournir des services. Le rapport de la NRC montre que les chemins de sont en fait fragiles et que le processus peut s'interrompre dans une des deux «vallées de la mort ». Pour la NRC, l'enjeu est donc de rendre les chemins de transitions plus rapides et plus efficace.

La première, la plus classique, concerne le passage d'un satellite de recherche à une série de satellites opérationnels. Elle renvoie à la difficulté à démontrer l'intérêt d'une nouvelle mesure pour une communauté d'utilisateurs, condition absolument nécessaire pour obtenir le financement des satellites opérationnels. La seconde, plus originale, correspond à une situation dans laquelle des satellites opérationnels produisent des données mais ces dernières ne sont pas ou peu utilisées en pratique. C'est précisément cette seconde vallée qui a été si difficile à franchir pour l'emploi des luminances satellitaires en prévision numérique du temps. En effet, les premières tentatives d'assimilation, à la fin des années 70, donnent des résultats décevants. Tracton et al. (1980) montrent ainsi que ces données n'améliorent pas la qualité de la prévision sur l'hémisphère nord ${ }^{3}$. Cette incapacité des données satellitaires à démontrer leur efficacité

\footnotetext{
${ }^{3}$ En matière de PNT, il est important de distinguer hémisphère nord et hémisphère sud. En effet, les données satellitaires ont été immédiatement utiles pour améliorer la qualité des prévisions dans l'hémisphère sud dans lequel les autres outils de mesures sont rares voire inexistant. Cet état de fait était problématique car $90 \%$ de la population mondiale, ainsi que les principaux financeurs de ces satellites au premier rang desquels on trouvait à l'époque les États-Unis, se situent dans l'hémisphère nord .
} 
pour la PNT est à l'origine d'un débat virulent entre la Nasa, en charge des satellites de recherche, et la Noaa qui finance et opère les satellites opérationnels. En effet, au sein de cette dernière le département en charge de la PNT (Noaa/NWS) en vient à adopter une position radicale rejetant en bloc les données spatiales dans l'hémisphère nord et préférant s'appuyer sur les données traditionnelles fournies par les stations au sol et les ballons, les satellites étant utilisés uniquement pour l'hémisphère sud pour lequel les données conventionnelles disponibles sont rares. La conséquence de ce conflit est immédiate : en 1982 la Nasa et la Noaa décident de geler les programmes de développement de nouveaux capteurs. Par conséquent pendant 20 ans les satellites vont voler avec des instruments, les sondeurs Tovs, conçus en 1978 (sur cet épisode voir plus particulièrement Conway, 2008). Les difficultés à assimiler leurs données persistent en effet. Ainsi 10 ans après l'article de Tracton et al., Andersson et al. (1991) démontrent que non seulement ces données n'améliorent pas la prévision mais qu'elles ont même tendance à la dégrader (voir également Pailleux (1996) pour une analyse fine de l'utilisation des luminances en PNT). La crise est donc totale, générant un malaise dans la communauté des météorologues. Comme l'explique Philippe Courtier, les satellites sont, de par leur couverture, un outil d'avenir pour la PNT et «c'est terrible de savoir que quelque chose est l'avenir et de ne pas savoir l'utiliser » (entretien, juin 2014). Pour savoir comment cette crise a été surmontée, un détour par les méthodes d'assimilation de données est nécessaire (encadré 1). C'est en effet à ce niveau que se situe le nœud du problème.

\section{L'assimilation de données}

En prévision numérique du temps ,l'assimilation de données est une méthode mathématique à laquelle on associe un algorithme permettant de définir les conditions initiales de l'atmosphère nécessaire au modèle. Cet algorithme combine de manière optimale diverses sources d'informations sur l'état de l'atmosphère recherché. Ce sont principalement les mesures météorologiques de natures variées (ballons sonde, stations sol, avions, bateaux, satellites, ...) et une ébauche (ou information a priori, c'est-à-dire indépendante des observations que l'on considère) généralement fournie par une prévision à courte échéance du modèle de prévision. Le critère d'optimalité pour cette condition initiale (ou analyse) est défini de manière statistique le plus souvent comme un minimum de variance (c'est à dire ayant la plus petite erreur possible). Pour satisfaire ce critère, il faut alors connaître les erreurs des diverses sources d'information : celles des observations (erreurs instrumentales, de représentativité, ...) et celles de l'ébauche. De manière classique, elles sont supposées de nature gaussienne avec une moyenne nulle. Elles sont dont caractérisées par une matrice de covariance d'erreurs d'observations (classiquement notée $\mathbf{R}$ ) et une matrice de covariances d'erreurs d'ébauche (classiquement notée B). La matrice B incorpore également d'autres informations sur les propriétés de l'écoulement atmosphérique comme l'équilibre hydrostatique ou l'équilibre géostrophique. L'assimilation de données conduit donc à estimer un état de l'atmosphère sur la grille structurée d'un modèle numérique à partir d'observations hétérogènes dans le temps et dans l'espace. Il s'agit donc d'une méthode d'interpolation avec des propriétés de filtrage fournies par la matrice B. Lorsqu'on cherche l'analyse comme une combinaison linéaire de l'ébauche et des observations on parle d'interpolation optimale (version simplifiée du filtrage de Kalman). Lorsqu'on cherche l'analyse comme un état minimisant une fonction coût représentant la somme de l'écart quadratique à l'ébauche et de l'écart quadratique aux observations on parle d'assimilation variationnelle. Lorsqu'on cherche un état de l'atmosphère à un instant donné on parle d'assimilation tridimensionnelle $(3 D)$, lorsqu'on cherche un ensemble d'états de l'atmosphère sur la fenêtre temporelle où les observations sont disponibles on parle d'assimilation quadridimensionnelle (4D). Généralement les approches 4D font évoluer la matrice B dans le temps. 


\section{La notion de dominant design}

Pour comprendre le problème, il est utile d'introduire le concept de dominant design. Ce concept, proposé à la fin des années 1970 par Abernathy et Utterback (1978), vise à traduire la stabilisation des caractéristiques d'un objet et des méthodes pour le concevoir ${ }^{4}$. En se basant sur l'analyse historique de plusieurs innovations, ces auteurs montrent en effet qu'à la suite de l'introduction d'une innovation, on assiste d'abord à un foisonnement des concepts autour de cette innovation, puis à une stabilisation des caractéristiques du produit. Les débuts de l'automobile ont ainsi vus une prolifération de concepts de voiture propulsées à la vapeur, à l'électricité, au gaz, à l'essence, avec 3 ou 4 roues, etc. Ce n'est que dans les années 1920 que l'automobile va trouver son dominant design qui perdure jusqu'à aujourd'hui: 4 roues, un moteur à explosion, une architecture monocorps acier, etc. (Geels, 2005). La littérature en management de l'innovation montre alors à la fois l'extraordinaire efficacité du dominant design pour concevoir et fabriquer une immense variété de produits toujours plus performants, et la difficulté à sortir de ce dominant design en raison de la standardisation des pratiques de conception qu'il induit (Henderson et Clark, 1990).

Outillés de ce concept revenons-en maintenant à notre histoire. En matière d'assimilation de données, il est en effet possible d'identifier, dans les années 1980, un dominant design que l'on peut caractériser en 3 points :

- En matière d'instruments il utilise des instruments de mesures qui produisent localement des mesures extrêmement précises (stations sol, ballons ${ }^{5}$, avions, etc.).

- Il s'agit donc de mesures synoptiques (c'est-à-dire relevées à heures fixes) et conventionnelles, qui mesurent directement le paramètre observé (par exemple la température à l'aide d'un thermomètre).

- Ces données sont assimilées par des méthodes d'estimation statistique appelées interpolation optimale (OI dans la suite, voir encadré 1). Comme l'explique E. Kalnay, responsable de la PNT à la Noaa de 1987 à 1997, cette méthode « est devenue la méthode opérationnelle dominante pendant les années 80 et au début des années 90 » (Kalnay, 2003.

\section{La crise de l'interpolation optimale}

Or c'est précisément là que se situe le problème. Le dominant design de l'OI entre en effet en crise dans les années 1980. Il rencontre plus spécifiquement trois difficultés. La première est son incapacité à traiter de manière dynamique l'évolution de l'incertitude pendant la prévision à court terme servant à construire l'ébauche (voir encadré 1). Dans cette méthode en effet, la matrice des covariances d'erreur d'ébauche (dite matrice B) est figée au début de la prévision et n'évolue plus ensuite. C'est ce problème qui intéresse en premier lieu les chercheurs en PNT dont fait partie Olivier Talagrand. Il s'agit là d'une question théorique générique qui n'a rien à voir avec les données satellitaires. Le second problème est le décalage croissant entre la

\footnotetext{
${ }^{4}$ On pourrait rapprocher cette notion de celle de paradigme, bien connue en philosophie des sciences, mais discuter ce rapprochement sortirait du cadre de cet article. Nous avons conservé le terme anglais car c'est lui qui est utilisé dans la littérature francophone.

${ }^{5}$ Qui va produire un profil de température, d'humidité et de vent à la verticale du lieu où il a été lâché (si on néglige la dérive du ballon au cours de son ascension).
} 
sophistication des modèles, dont la résolution notamment a considérablement augmenté, et les méthodes d'assimilation qui déterminent la condition initiale de la prévision. Comme l'explique François-Xavier Le Dimet (septembre 2014), à cette époque l'assimilation est devenue «le point faible de la prévision (...) parce que la condition initiale qu'on obtenait avec l'interpolation n'était pas suffisante en qualité ». C'est en grande partie ce qui explique les résultats d'Andersson et al. (1991). Enfin, troisième difficulté, l'OI n'arrive pas à intégrer de manière satisfaisante les données satellitaires ${ }^{6}$. Or celles-ci, de par leur couverture, constituent «sans contestation possible» (Philippe Courtier, juin 2014) la solution pour obtenir les données globales nécessaires à la prévision, mais coûtent cher. Cette crise de l'OI se traduit à l'époque par une stagnation des performances de la prévision. Woods (2005), dans son histoire du CEPMMT, note ainsi que, dans les années 1980, «il semble qu'un plateau ait été atteint dans la précision des prévisions du centre. (...) D. Burridge [directeur de la recherche] avait le sentiment que le système d'assimilation par interpolation optimale avait été poussé à ses limites. Les nombreuses données satellitaires n'étaient pas utilisées de manière optimale. Quelque chose devait donc être modifié, mais quoi ?".

\section{Les données spatiales : une rupture technique}

Pour comprendre la difficulté de l'assimilation des données satellitaires, un détour technique est nécessaire. Le problème est lié à la fois à la nature des données spatiales et aux méthodes utilisées à l'époque pour les assimiler. En effet, par rapport aux mesures de température effectuées par les ballons sondes, les données produites par les satellites constituent une rupture très importante : elles sont indirectes. Autrement dit, un sondeur atmosphérique ne mesure pas directement la température de l'atmosphère. Il mesure des luminances dont il va être possible, après inversion, d'extraire la température (Eyre et al., 1993). La difficulté ici vient de la nature de la relation entre luminance et température telle qu'exprimée par la fonction de transfert radiatif (figures 2 et 3 ).

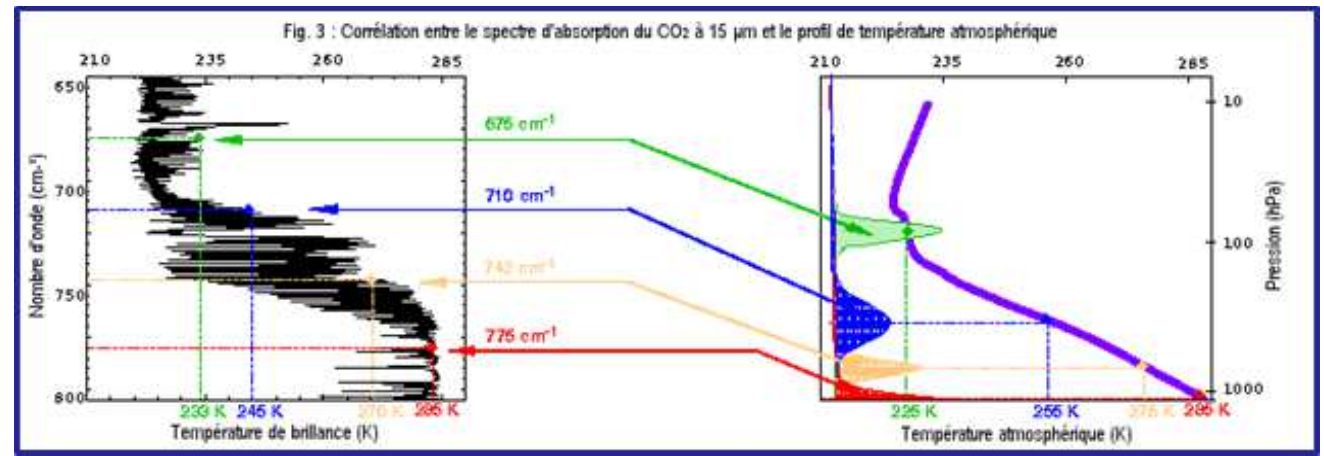

Figure 2 : Représentation schématique de la correspondance entre luminances et températures Source : https://iasi.cnes.fr/fr/IASI/Fr/GP_mission.htm.

\footnotetext{
${ }^{6}$ Pour être tout à fait exact dans les années 1980, Y. Durand avait réussi à utiliser directement les luminances dans le modèle régional Peridot de Météo-France mais au prix d'une complexité algorithmique qui rendait difficilement transférable la méthode (entretien avec Philippe Courtier, juin 2014, voir également Pailleux, 1996).
} 
La fonction de transfert radiatif (figure 3) permet de passer du profil de température (à droite) à la mesure du satellite (à gauche)

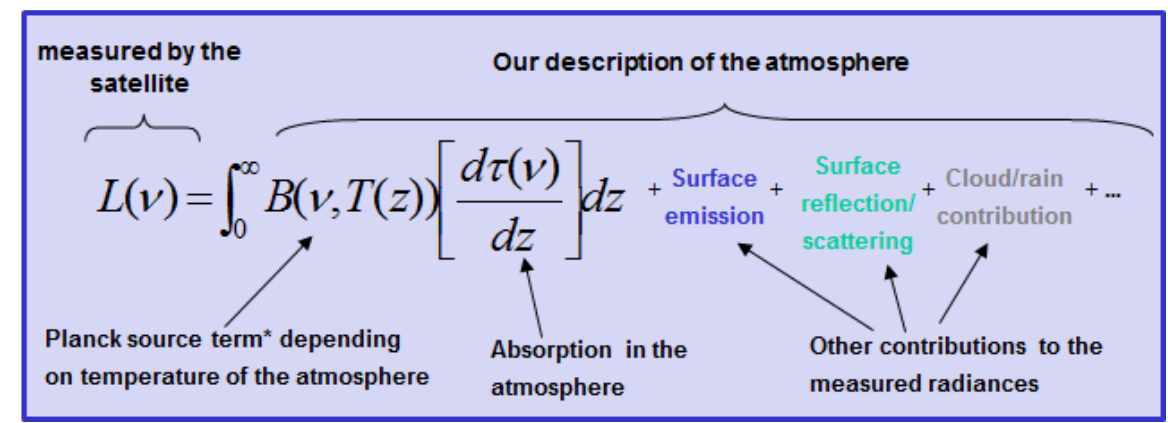

Figure 3. La fonction de transfert radiatif au CEPMMT

Source : https://earth.esa.int/documents/973910/997417/TM_1.pdf .

Ainsi comme l'explique Jean Pailleux (juin 2014), les météorologues se sont retrouvés confrontés à « un problème inverse typique. On mesure y [luminances] qui a l'équivalent de 2 à $3 \%$ d'info et on cherche à reproduire $x$ et $z$ [humidité et température] qui est une information beaucoup plus riche. Mais il se trouve que la fonction de transfert radiatifn'est pas inversible. On sait très bien calculer les luminances quand on a la température et l'humidité, mais quand on a quelques valeurs de luminances on n'arrive pas à restituer $x$ et $z$ sans informations auxiliaires ». En conséquence, la solution initiale a consisté à produire des profils de température et d'humidité similaires à ceux mesurés par les ballons sondes à partir des données spatiales. Comme l'explique le Directeur de la prévision de la Noaa en 1969, "si vous pouvez les faire ressembler à des radiosondages, nous pourrons les utiliser" (NRC, 2003). Cette première stratégie, qualifiée de «satellite-to-model» par Hollingsworth (1990), visait à faire correspondre les données spatiales aux exigences du modèle. À l'époque, ce travail était fait par la Noaa, seul organisme disposant de satellites météorologiques opérationnels en orbite polaire. À l'aide de méthodes statistiques, les météorologues américains fabriquaient ainsi des «pseudo-sondages » appelés SATEM. Or les techniques utilisées à l'époque conduisaient à un appauvrissement considérable de l'information produite par les satellites. Comme l'explique Jean Pailleux (juin 2014), ces SATEM «avaient l'équivalent de deux ou trois points sur la verticale en température. Donc ils indiquaient simplement si la stratosphère était plus chaude que la troposphère, s'il y avait un gradient de température normal. C'était une information très très pauvre (...) par rapport aux ballons et par rapport aux besoins des modèles de prévision numériques qui avaient déjà 10, 15, 20 niveaux. (...) De plus, quand on regardait l'info réelle de ce sondage, on s'apercevait qu'il contenait un peu d'informations provenant du sondeur luimême et beaucoup d'informations venant d'un fichier climatologique ou d'un modèle de prévision américain. Donc c'était une information qui n'était pas de l'information satellitaire pure et qui était contaminée par de l'information auxiliaire. » (voir aussi Pailleux, 1996). Sans surprise, l'assimilation de ces pseudo-sondages dans des modèles de prévision de plus en plus sophistiqués n'améliorait pas la qualité de la prévision dans l'hémisphère nord, voire la dégradait. Cette première approche consistant à insérer les nouvelles données (approximées) dans les algorithmes d'assimilation basés sur l'interpolation optimale a donc abouti à des 
résultats décevants en termes d'amélioration de la PNT. Comment cette crise a-t-elle été surmontée?

\section{L'émergence des méthodes variationnelles en assimilation}

En fait, comme nous l'avons dit, l'OI comporte d'autres limites que celles de l'assimilation des données spatiales, en particulier la non prise en compte de l'évolution de l'incertitude pendant la prévision. Le développement de méthodes d'assimilation alternatives constitue par conséquent un sujet de recherche pour les météorologistes. Ses racines sont même anciennes. Ainsi, dès 1955, Yoshikazu Sasaki, à l'université d'Oklahoma, a proposé d'utiliser les méthodes d'assimilation dites «variationnelles ». Ses travaux restent toutefois très théoriques, sans applications concrètes et sans beaucoup d'écho. En effet, dans l'assimilation variationnelle, les méthodes d'estimation de l'interpolation optimale sont remplacées par la minimisation d'une fonction de coût qui mesure l'écart entre le modèle et l'ensemble des informations disponibles au moment de la prévision, c'est-à-dire les observations et l'ébauche fournie par la prévision précédente (Philippe Courtier, mai 2014 ) $^{7}$. Rabier et al. (2000) décrivent ainsi le principe du 4D-VAR (Figure 4) : «Sur la fenêtre temporelle de six heures centrée sur 12 h UTC, la prévision effectuée à partir de l'ébauche $X_{b}$ à 9 h UTC, avant prise en compte des données d'observation, décrit six heures d'évolution temporelle des champs atmosphériques (trait rouge). La prévision corrigée part de l'état initial $X_{a}$ qui a été modifié pour ajuster la nouvelle trajectoire en fonction des observations disponibles dans la fenêtre d'assimilation (trait bleu). La fonction de coût que l'on a ainsi minimisé fait intervenir, d'une part, la distance entre $X_{b}$ et $X_{a}$ (terme $J_{b}$ d'écart à l'ébauche) et, d'autre part, les distances entre la succession d'états atmosphériques issus de $X_{a}$ et les observations réparties dans le temps (terme $J_{o}$ d'écart aux observations). » (Rabier et al., 2000, p. 90). Or c'est là que les difficultés apparaissent. En effet, la minimisation de cette fonction de coût, qui intègre dans sa boucle de résolution le modèle de prévision lui-même, est extrêmement coûteuse en temps de calcul, en raison notamment de la dimension gigantesque du problème à résoudre ${ }^{8}$. Par conséquent, comme l'explique Philippe Courtier, la méthode, dans son principe, est au-delà de la puissance de n'importe quelle machine de l'époque. C'est ce verrou qu'il va falloir réussir à faire sauter.

\footnotetext{
${ }^{7}$ En fait, deux variantes de la méthode sont envisageables : dans le 3D-Var, cette minimisation a lieu une fois au début de la prévision, alors que le 4D-Var intègre aussi la dimension temporelle. Dans ce dernier cas, la méthode « ne vise pas à obtenir l'état optimal à un instant donné, mais la trajectoire optimale sur une fenêtre de temps donné. Les observations sont donc prises en compte aussi bien dans leur distribution spatiale que temporelle. (...) Le 4D-Var apporte un aspect temporel car il propage l'information apportée par les observations à l'instant initial de la fenêtre d'assimilation. De ce fait, l'analyse obtenue doit permettre au modèle d'évolution d'avoir la trajectoire la plus proche possible de l'ensemble des observations utilisées. Cette amélioration du 3D-Var permet d'ajouter la connaissance de l'évolution du système comme information pour l'analyse » (Daget, 2007). Voir la figure 4 ainsi que Rabier et al. (2000).

${ }^{8}$ De l'ordre de 50 millions pour la variable définissant l'état d'un modèle numérique dans les années 1990.
} 


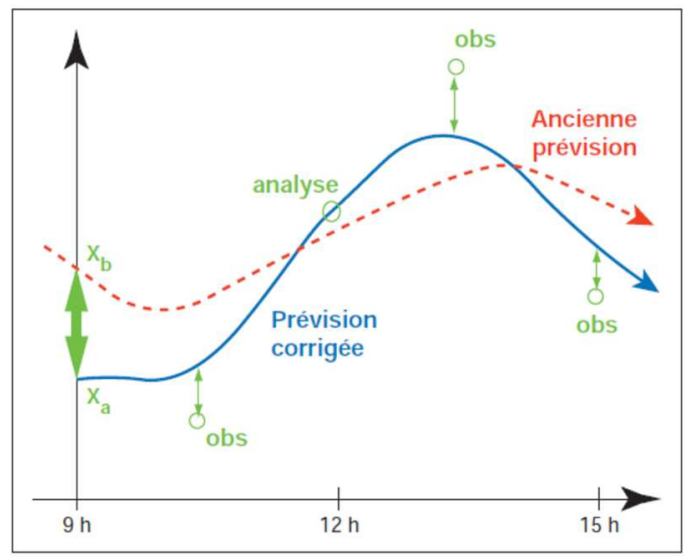

Figure 4. Schéma décrivant le principe de l'assimilation variationnelle quadridimensionnelle (4D-Var) dans Rabier et al. (2000)

D'autres chercheurs s'intéressent d'ailleurs à cette question de l'assimilation des données. Au Laboratoire de météorologie dynamique, Olivier Talagrand développe dans sa thèse d'État et dans des articles (Talagrand, 1981a, 1981b) d'autres méthodes d'assimilation, elles aussi très éloignées des applications opérationnelles. De son coté, François-Xavier Le Dimet, professeur de mathématiques appliquées à l'université de Clermont-Ferrand, s'intéresse également à la météorologie et aux méthodes variationnelles. C'est un spécialiste du contrôle optimal, proche du célèbre mathématicien Jacques-Louis Lions. Il a fait sa thèse sur la modélisation des processus stochastiques pour simuler la croissance et l'interaction des gouttes d'eau dans les nuages. Intéressé par les applications du contrôle optimal et les méthodes variationnelles en météorologie, il a travaillé en 1982 avec Yoshikazu Sasaki. Il apporte notamment ses compétences en contrôle optimal que les météorologues d'Oklahoma ne connaissent pas. Un premier article est ainsi soumis à la revue Tellus, référence dans le champ. Il est rejeté avec toutefois les encouragements d'un des relecteurs. Arrive alors une rencontre importante. En 1983, un congrès de la Société française de physique sur la physique de l'atmosphère se tient à Clermont-Ferrand. C'est l'occasion d'une rencontre qui va s'avérer déterminante avec Olivier Talagrand. Ce dernier connait très bien les problèmes opérationnels des météorologues et ses connaissances mathématiques sont suffisamment solides pour comprendre ce que fait Le Dimet. Il a notamment déjà entendu parler des techniques adjointes via ses contacts avec des mathématiciens russes. Comme il explique (14 septembre 2014) : "J'ai étudié dans ma thèse [1977] une méthode d'assimilation un peu simpliste (mais je ne savais pas comment faire autrement). Et ce n'est que quand j'ai rencontré François-Xavier Le Dimet que j'ai compris comment faire ce qui me semblait souhaitable. Le problème n'étant pas tant l'idée variationnelle, assez simple en soi, que sa mise en cuvre numérique dans des systèmes de grande dimension. C'est la technique des équations adjointes qui a fourni la clé du problème. Cela étant, tout cela était bien loin de l'utilisation des données spatiales dans les modèles de prévision météo. ». Techniquement parlant le problème majeur est en effet de calculer le gradient de la fonction de coût par rapport à l'état d'un modèle de très grande dimension (qui équivaut à la dérivée ou « la direction de plus forte pente », Philippe Courtier, juillet 2014). Or, compte tenu justement de la dimension du problème, le calcul du gradient était jusque-là impossible ce qui bloquait le développement des méthodes variationnelles. Les méthodes du contrôle optimal apportées par Le Dimet, en particulier la technique dites des équations 
adjointes ${ }^{9}$, vont permettre de lever ce verrou. La rencontre entre Le Dimet et Talagrand aboutit ainsi à un article fondamental pour l'assimilation publié en 1986 dans la revue Tellus. Intitulé «Variational algorithms for analysis and assimilation of meteorological observations: Theoretical aspects », il est aujourd'hui cité plus de 1700 fois ${ }^{10}$. On peut considérer qu'il est à l'origine de l'explosion des travaux sur les méthodes variationnelles que l'on peut observer dans la revue de littérature proposée par Courtier et al. (1993) et montrée sur la figure $5^{11}$.

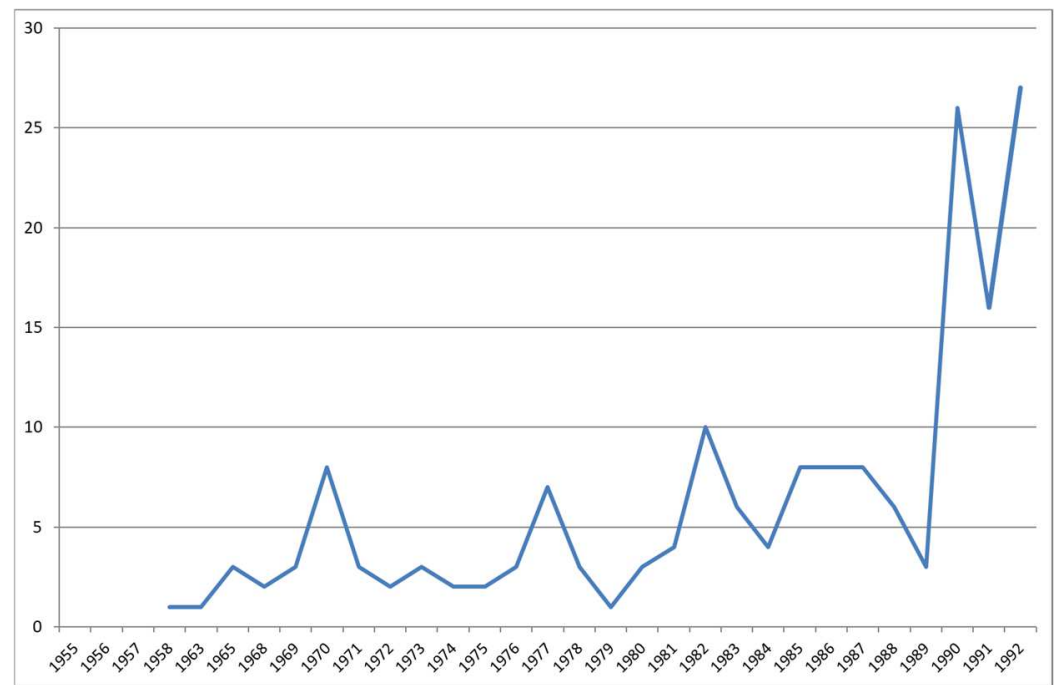

Figure 5. Évolution du nombre de publications sur l'assimilation variationnelle et les filtres de Kalman sur la période 1955-1993 d'après Courtier et al. (1993).Selon Lenfle (2015).

\section{La longue route vers la mise en ouvre opérationnelle}

En effet, une fois cette étape franchie, Olivier Talagrand passe immédiatement à l'étude des potentialités opérationnelles de la nouvelle méthode en collaboration avec un de ses doctorants, Philippe Courtier. Ce dernier effectue sa thèse sous sa direction sur L'application $d u$ contrôle optimal à la prévision numérique en météorologie. Elle est soutenue en 1987 devant un jury présidé par Jacques-Louis Lions. La thèse et les articles qui en découlent (Talagrand et Courtier, 1987 ; Courtier et Talagrand, 1987, 1990) démontrent l'intérêt et la faisabilité de l'assimilation variationnelle pour la prévision numérique, d'abord avec des modèles simples (à une couche dans les articles de 1987) puis avec un modèle à trois dimensions (dans l'article de 1990). L'assimilation de données devient alors un sujet majeur pour la communauté des météorologues. Le Dimet et Talagrand organisent ainsi, à la demande de l'Organisation

\footnotetext{
${ }^{9}$ Il n'est pas question ici de rentrer dans la technique du contrôle optimal et des méthodes adjointes, ce dont nous serions d'ailleurs bien incapables. Le lecteur intéressé se réfèrera à Le Dimet et Talagrand (1986), Talagrand (1997), Kalnay (2003), Daget (2007) ou, sur les méthodes adjointes, Errico (1996).

${ }^{10}$ Notons également qu'en 2012 François-Xavier Le Dimet a été élu Fellow de l'American Meteorological Society pour ses travaux pionniers en assimilation de données.

${ }^{11}$ Précisons que Lewis et Derber ont publié en 1985 un papier qui lui aussi utilise la technique des équations adjointes. Toutefois, ils précisent clairement dans les remerciements que l'idée leur a été donnée par Le Dimet et Talagrand au cours d'une rencontre au Canada, ce que les auteurs nous ont confirmé lors des entretiens. Ainsi John Derber reconnaît la contribution fondamentale de l'article de 1986 : «Pour utiliser directement les luminances, il faut commencer par développer le système variationnel. Dans la forme proposée par Sasaki, il était difficile de voir comment cela pouvait être mis en pratique. Avec Le Dimet et Talagrand, c'est devenu faisable » (entretien John Derber, septembre 2014).
} 
météorologique mondiale, le premier colloque international sur l'assimilation de données en météorologie et océanographie à Clermont-Ferrand en juillet $1990^{12}$. Comme l'explique Le Dimet (septembre 2014), « cela correspondait vraiment à une demande (...) parce qu'il y avait ce trou dans la prévision qui faisait qu'il était inutile d'améliorer les modèles si l'on n'était pas capable de déterminer plus précisément les conditions initiales ». Or l'intérêt des méthodes variationnelles est précisément de dépasser les limites de l'interpolation optimale en vigueur qui, «ne tient compte grosso modo que des données et des statistiques, tandis que quand vous utilisez du contrôle optimal, vous forcez la condition initiale à être conforme à la physique du modèle. La condition initiale que vous obtenez a vraiment une signification physique» notamment car la fonction de coût que l'on cherche à minimiser fait intervenir explicitement les équations d'évolution du modèle de PNT dans un formalisme quadridimensionnel..

Une des caractéristiques importantes de cette histoire est que ces recherches n'ont pas été entreprises spécifiquement pour mieux assimiler les données spatiales. L'enjeu est d'abord de renforcer les fondements conceptuels et mathématiques de l'assimilation. La question va toutefois assez rapidement devenir importante en raison des difficultés persistantes à intégrer les données satellitaires qui sont incontournables mais particulièrement coûteuses en raison des coûts du satellite et de son lancement. L'assimilation variationnelle va en effet apporter «une méthode tout à fait systématique pour introduire dans le processus d'assimilation n'importe quelle source d'information »(Olivier Talagrand, septembre 2014). De fait la montée en puissance de cette question apparaît dans les actes du colloque de Clermont-Ferrand. Une des deux conférences inaugurales, faite par Anthony Hollingsworth, alors directeur de la division d'assimilation de données du CEPMMT, s'intitule «Assimilation of remotely sensed atmospheric data from new satellite system in the 1990s ». Face aux difficultés rencontrées, la question de l'assimilation des luminances est en effet devenue, sous l'impulsion de John Eyre, un axe de recherche important au CEPMMT et au Met Office britannique. La logique, à la fin des années 1980, reste encore toutefois de produire des profils de température que l'on va insérer dans le modèle (satellite-to-model) mais en utilisant la méthode variationnelle pour l'extraction, ce qui permet de maitriser les incertitudes associées aux données satellitaires (Thépaut et Moll, 1990 ; Eyre et al., 1993). Même si Courtier qualifie l'impact de «très légèrement positif mais un peu décevant», ces travaux montrent la faisabilité d'une approche variationnelle et ouvrent la voie au 3D et au 4D-Var pour l'assimilation des données spatiales. Cette méthode va en effet permettre de rompre avec la logique d'inversion des luminances (satellite-to-model) dont nous avons vu précédemment les limites. Ainsi, en 3D/4D-Var on introduit un opérateur d'observation dont le rôle est de «transformer une variable géophysique en une quantité directement comparable à une mesure instrumentale » (Jean-Noël Thépaut, mars 2015). Autrement dit, cet opérateur d'observation va permettre, à partir de variables du modèle, typiquement la température et l'humidité, de générer des luminances que l'on va ensuite pouvoir comparer à ce qui est mesuré par le satellite. Dans le cas qui nous intéresse l'opérateur d'observation correspond à la fonction de transfert radiatif. La logique des inversions

\footnotetext{
${ }^{12}$ Nous tenons à remercier François-Xavier Le Dimet pour nous avoir transmis un exemplaire des actes de ce premier colloque qui réunit, autant que nous puissions juger, toute la communauté des acteurs de la météo concernés par l'assimilation des deux côtés de l'Atlantique (Talagrand, Courtier, Le Dimet, Eyre, Hollingsworth, Pailleux, Sasaki, Derber, Thépaut, Lorenc, Mahfouf, etc).
} 
(retrievals) est ainsi abandonnée au profit d'une approche qui va, cette fois, du modèle vers le satellite (model-to-satellite dans Hollingsworth, 1990). Comme l'explique Jean Pailleux (juin 2014), «on va partir de la température et de l'humidité et on va calculer la luminance «modèle » et on va voir si la luminance «modèle » colle avec la luminance «observée » qui résulte directement de la physique du rayonnement. C'est presque ce que produit l'instrument radiomètre. (...) on n'a donc plus besoin d'inverser directement la fonction de transfert radiatif qui, de toute façon, ne l'est pas ». Toutes les hypothèses nécessaires à la procédure d'inversion sont alors connues, ce qui va permettre, enfin, d'exploiter pleinement l'information produite par les satellites.

Reste que la rupture conceptuelle que constitue l'assimilation variationnelle ne représente que le début de l'histoire. En cette fin des années 1980, la route est encore (très) longue jusqu'à la mise en œuvre opérationnelle de la méthode. Plusieurs difficultés identifiées constituent autant d'obstacles à franchir.

- La mise en œuvre des techniques du contrôle optimal sur des systèmes aussi complexes que les modèles de PNT n'est pas triviale. Il ne s'agit aucunement d'un simple transfert de techniques mises au point ailleurs. La dimension du des modèles de PNT et le caractère non-linéaire des phénomènes sous-jacents n’ont de fait jamais été étudiés par les tenants du contrôle optimal.

- La possibilité de mise en œuvre de la méthode est, comme c'est toujours le cas en météorologie, étroitement liée à l'évolution de la puissance des calculateurs. La méthode variationnelle, en particulier dans sa forme 4D est, comme expliqué précédemment, intrinsèquement extrêmement coûteuse en temps de calcul. Comme l'explique FrançoisXavier Le Dimet (septembre 2014), «en 1982 quand on a publié les premiers articles, c'était absolument impossible. (...) Sans évolution du calcul ce sont des méthodes qui n'avaient pas de sens. On ne peut pas isoler les méthodes. Il faut mettre ça dans la perspective de l'évolution des moyens de calcul. Sans la puissance de calcul c'était une très très mauvaise idée ». Il va donc être nécessaire d'une part de développer des méthodes, dont l'adjoint fait partie, permettant de réaliser les calculs dans les délais extrêmement courts de la PNT et, d'autre part, de coordonner la mise en œuvre avec l'évolution des calculateurs. Le problème est d'autant plus compliqué que, dans le même temps, le modèle de prévision évolue lui aussi et consomme le temps de calcul disponible, ce qui fait que l'on est toujours à la limite des performances des calculateurs.

- Reste enfin l'énorme travail de mise en place dans des systèmes opérationnels avec ce que cela suppose de codage, validation, débogage, contrôle de la qualité des données, etc. mais aussi résistance des opposants. Olivier Talagrand (septembre 2014) est extrêmement clair sur la difficulté qu'il y a à insérer une nouvelle approche dans un système opérationnel existant: «Les modèles météo étaient déjà à l'époque, des machines immenses, des logiciels qui ont une très grande inertie. (...) Et nous nous sommes trouvés face à un problème technique auquel apparemment les gens de l'école de Lions n'avaient jamais été confrontés car quand ils utilisaient la méthode adjointe, il n'y avait pas de modèle numérique préexistant. Il y avait un problème et au niveau des codes, ils écrivaient le 
modèle direct et l'adjoint ensemble. Jamais personne ne s'était trouvé devant un énorme code dont il fallait prendre l'adjoint. ».

On comprend mieux dès lors, la boutade de François-Xavier Le Dimet (septembre 2014) nous disant en riant : "Si j'avais su [en 1982-83] ce que ça coûtait [en capacité de calcul], j'aurais abandonné tout de suite (...) On ne soupçonnait pas les difficultés qu'il y avait [dans la mise en œuvre opérationnelle]».

\section{Le projet IFS/Arpege}

La tâche et les enjeux sont tels qu'un projet commun au CEPMMT et à Météo-France est décidé et lancé formellement en octobre 1988 lors d'une réunion commune à Reading (Pailleux et al., 2015). Comme l'explique Jean-Noël Thépaut (septembre 2014), «c'était un chantier gigantesque il fallait tout développer les modèles adjoints, les modèles linéaires tangents et, en parallèle, commencer à s'intéresser aux données satellitaires parce que c'était une des justifications de l'approche variationnelle ». La tâche suppose donc des moyens qui dépassent complètement une équipe de recherche. Il s'agit de fait d'une décision très risquée. Ainsi pour Jean-Noël Thépaut, « il fallait être un peu visionnaire quand même parce que quand Talagrand et Courtier ont sorti leur papier avec le modèle très simple d'équations en eau peu profonde, les moyens de calcul nécessaires n'existaient pas. Et il fallait être assez courageux pour anticiper qu'ils seraient disponibles parce qu'on multipliait le temps de calcul par rapport à l'OI de manière colossale. ». Et de fait, en 1988, rien ne garantit la faisabilité opérationnelle de la méthode.

Notre objectif ici n'est pas de reconstituer dans le détail l'organisation et l'histoire du projet IFS/Arpege ${ }^{13}$ (voir les grandes dates du projet dans l'encadré $n^{\circ} 2$ ). Nous souhaiterions ici insister sur l'ampleur de la tâche consistant à introduire une nouvelle méthode d'assimilation de données, en rupture complète avec le dominant design, dans un système opérationnel. Cette dernière contrainte est en effet majeure par ce qu'elle induit en termes de transmission de données, de contrôle qualité, de vitesse de calcul, etc. IFS/Arpege va ainsi mobiliser environ 10 personnes équivalents temps plein (ETP) pendant 10 ans, ce qui est considérable.

\footnotetext{
${ }^{13}$ Le lecteur cherchant à connaître les grandes étapes du projet se réfèrera à Andersson et Thépaut (2008) ou, plus récemment Pailleux et al. (2015)
} 
- Juillet 1987 : Début de la phase de développement

- Octobre 1988 : Lancement officiel du projet IFS/ARPEGE. La mise en place du 4D-VAR est alors prévue en 1993

- 1991 : Décalage de la date de mise en place opérationnelle à fin1995-96 en raison des coûts de calcul, de la nécessité de développements supplémentaires sur le logiciel, la science et les algorithmes.

- 2 mars 1991 : Mise en œuvre du modèle IFS sur le supercalculateur CRAY C90 du CEPMMT

- 30 janvier 1996: Mise en œuvre opérationnelle du 3D-VAR au CEPMMT.

- 19 septembre 1996 : Le 3D-VAR migre du CRAY vers un supercalculateur FUJITSU. L'OI, qui n'avait pas été adaptée au FUJITSU, est définitivement abandonnée au CEPMMT.

- 1997 : Mise en œuvre opérationnelle du 3D-VAR à Météo-France et du 4DVAR à l'ECMWF (25 novembre )

- 20 juin 1999: Mise en œuvre opérationnelle du 4D-VAR à Météo-France.

Sans entrer dans le détail du déroulement du projet, notre recherche nous a conduit à identifier trois éléments qui expliquent une large part de «la sueur et des larmes » (Jean-Noël Thépaut) pendant les 10 années de travail nécessaires.

1. Le recodage du modèle de prévision. La première difficulté à laquelle a été confrontée l'équipe projet a été celle du développement d'un nouveau modèle de prévision afin d'en générer l'adjoint (transposée de sa version linéarisée) en même temps. Comme l'explique Olivier Talagrand, «Jamais personne ne s'était trouvé devant un énorme code dont il fallait prendre l'adjoint. Une difficulté typique c'est qu'il faut procéder à rebours. Supposons que j'aie dans un code un système de branchement, de type "if ", qui fait que je ne peux pas remonter. Il y en a partout dans les codes météos. Il fallait donc réécrire le code de telle façon qu'on puisse remonter au travers du code. Ça c'était une difficulté. Et lorsque Philippe Courtier est allé au CEPMMT pour mettre en ouvre l'assimilation variationnelle, après avoir étudié le problème et en avoir discuté avec les collègues, ils ont décidé que c'était une excellente occasion de réécrire le code du modèle en entier. ». Ce travail, qui va aussi permettre d'introduire des modifications dans l'architecture même du modèle, va prendre deux ans et mettre en pause les travaux sur l'assimilation, qui ont besoin du nouveau modèle pour être réalisés.

2. Le développement de la méthode incrémentale afin de réduire les coûts de calcul. L'autre énorme difficulté du projet IFS/Arpege concerne les coûts de calcul de la méthode variationnelle. Comme nous l'avons vu, le problème vient de la minimisation de la fonction de coût mesurant la distance du modèle aux observations et à l'ébauche, qui doit se faire sur un laps de temps réduit en raison des contraintes opérationnelles de la prévision. La rupture conceptuelle permettant de résoudre la question du coût de calcul va venir, comme l'expliquent Philippe Courtier et Jean-Noël Thépaut, d'une discussion à Reading avec John Derber de la Noaa (voir aussi Rabier et al.,, 2000). La 
méthode consiste schématiquement à réaliser les itérations nécessaires à la minimisation de la fonction de coût sur une version simplifiée, avec une maille plus large, du modèle de prévision tout en utilisant périodiquement («une fois toutes les 30 minimisations », Courtier, juillet 2014) le modèle complet pour recaler l'algorithme de minimisation. Cette approximation, qui dégrade la qualité («mais de toute façon on est coincé», Philippe Courtier, juin 2014), «permet de résoudre de façon raisonnable [le problème du coût de calcul]. (...) on gagne à peu près un ordre de grandeur en temps de calcul ». Le verrou que constituait le temps de calcul de l'assimilation variationnelle est alors levé. Comme l'explique Jean-Noël Thépaut (septembre 2014), «avec cette technique incrémentale, où la grille d'analyse est plus large que la grille du modèle, on fait des simplifications sur les modèles linéaire tangent et adjoint, [qui ont] permis de faire rentrer le 4D-Var sur le calculateur du centre européen ».

3. Et, enfin, le réglage de $B$, la matrice des covariances d'erreur d'ébauche. Malgré tous ses efforts, l'équipe IFS/Arpege n'est pas au bout de ses surprises, en particulier en ce qui concerne l'assimilation des données satellitaires. Nous sommes en 1992, Philippe Courtier est de nouveau au CEPMMT après être retourné à Météo-France. Le modèle a été recodé, la méthode incrémentale est en cours d'élaboration et l'on progresse vers la mise en œuvre opérationnelle du 3D-Var. L'intégration des luminances constitue alors un enjeu important du projet. Or les travaux réalisés en 1993 montrent que, malgré toutes ces améliorations, l'assimilation des luminances n'améliore toujours pas significativement la qualité de la prévision. L'explication du problème va venir de ce que l'on appelle les fonctions de structure qui sont contenues dans la matrice des covariances d'erreur d'ébauche, dite matrice $\mathbf{B}$, qui joue un rôle fondamental dans la qualité de la prévision ${ }^{14}$. Philippe Courtier (juin 2014) se souvient alors qu'en «1986 ou 1985, Anthony Hollingsworth et Peter Lönnberg (...) avaient réglé $\boldsymbol{B}$ vers la meilleure utilisation possible des AIREP, des données de vent que l'on a à peu près à $300 \mathrm{mb}$ grâce aux avions qui traversent l'Atlantique (ou le Pacifique). Et du coup, on utilisait moins bien l'information de température qui était en quelque sorte filtrée. Et c'est la raison pour laquelle l'impact du $1 D$-Var et du 3D-Var était demeuré marginal. On ne récupérait pas l'information de température présente dans les luminances. C'était une approximation qui favorisait le vent, pour de bonnes raisons car les données de vent étaient de bonne qualité et très précieuses, mais du coup on n'extrayait pas d'information de température des luminances ». Le changement du réglage de $\mathbf{B}$ va alors, enfin, permettre d'extraire des luminances l'information nécessaire à l'amélioration de la précision de la PNT.

Ce dernier point illustre selon nous la complexité de l'intégration d'une nouvelle donnée dans un système opérationnel qui fonctionne depuis une décennie sur d'autres bases théoriques et,

\footnotetext{
${ }^{14}$ Sur cette question voir par exemple Kalnay (2003) qui explique notamment que «la matrice des covariances d'erreur d'ébauche $\mathbf{B}$ constitue l'erreur de covariance la plus difficile à estimer, et a un impact crucial sur les résultats ».
} 
corollaire, le degré de précision des connaissances nécessaires pour le faire évoluer. Elle montre le chemin à parcourir pour passer d'un nouveau concept de traitement de la donnée, l'assimilation variationnelle, à la réalisation effective des promesses qu'il contient dans un système opérationnel. La diable est vraiment dans les détails et il est nécessaire de creuser très profond pour comprendre les processus à l'œuvre, les points bloquants qui peuvent, jusqu'au dernier moment, faire échouer le projet.

C'est finalement en janvier 1996 que le 3D-Var va être mis en œuvre opérationnellement au centre européen (Courtier et al., 1998) suivi en novembre 1997 par le 4D-Var (Rabier et al., 2000). C'est aussi en 1998 qu'est démontré, enfin serions-nous tentés de dire, l'impact positif de l'assimilation directe des luminances sur la qualité de la prévision dans l'hémisphère nord (Derber et $\mathrm{Wu}, 1998$ ) en utilisant le système 3D-Var mis en œuvre opérationnellement à la Noaa/NCEP en octobre 1995. Dix-sept années se sont écoulées depuis que la Noaa a produit les premiers pseudo-sondages opérationnels issus des sondeurs atmosphériques ${ }^{15}$. MétéoFrance basculera au 4D-Var en 1999 avant que d'autres centres de prévision suivent le mouvement ${ }^{16}$, marquant ainsi la diffusion de ces nouvelles méthodes d'assimilation (Bauer et al., 2015).

\section{Conclusion : de la valeur des données}

On peut finalement résumer cette histoire ainsi : l'apparition d'un nouvel instrument de mesure, le sondeur satellitaire, dans un dominant design de la PNT qui présentait par ailleurs un certain nombre de limites, a provoqué un changement de ce dominant design dans les méthodes d'assimilation de données. Ce basculement s'est fondé sur une rupture conceptuelle, l'assimilation variationnelle et les méthodes adjointes (Le Dimet et Talagrand, 1986). Sur ces bases théoriques renouvelées, le CEPMMT et Météo-France ont lancé un projet majeur, IFS/Arpege, aboutissant, 10 ans plus tard, à la mise en œuvre opérationnelle de la méthode au CEPMMT puis à Météo-France, et enfin dans les principaux centres de prévision.

Cette longue aventure permet de montrer la complexité de l'utilisation de données nouvelles, l'importance des investissements nécessaires et, par conséquent, la difficulté à franchir les vallées de la mort identifiées par le NRC. Elle permet aussi de remettre en cause un certain nombre d'idées reçues sur la valeur des données. En effet, l'hypothèse implicite souvent faite dans l'industrie spatiale nous semble être que la donnée a de la valeur en tant que telle. John Derber de la Noaa, qui a joué un rôle important dans cette histoire, nous expliquait ainsi en juillet 2014 se souvenir «d'un important scientifique de la Nasa nous donnant une présentation sur Airs avant son lancement. Il affirmait que comme l'instrument serait deux fois meilleur que

\footnotetext{
${ }^{15}$ Nous ne développerons pas ce point plus avant dans cet article mais il est important de noter qu'une des forces de l'assimilation variationnelle réside dans son caractère génératif. En effet, une fois mise en place, la méthode s'est révélée extraordinairement efficace pour intégrer une grande variété de données météorologiques (par exemple les données de radio-occultation GPS) et a trouvé des applications dans d'autres domaines (médecine, agronomie, urbanisme, etc) (voir Thépaut et Mahfouf, 2003). Comme l'explique François-Xavier Le Dimet (septembre 2014), la technique constitue en effet une réponse possible à une question générique : «comment coupler de l'information hétérogène en nature »?

${ }^{16}$ Ont ensuite suivi le mouvement le UK Met Office en 2004, la Japan Meteorological Agency et Environnement Canada en 2005 et le US Naval Research Laboratory en 2009.
} 
l'instrument HIRS ${ }^{17}$, cela se traduirait par une division de moitié de l'erreur de prévision». Une telle assertion est évidemment fausse. Nous venons au contraire de montrer d'une part la complexité intrinsèque des données spatiales comme les luminances et, d'autre part, que ces données n'ont pas de valeur sans les algorithmes qui permettent de les étalonner puis de les assimiler dans les modèles (voir également Cirac, 2014). Schématiquement c'est le couple luminances / assimilation variationnelle qui permet d'améliorer la PNT. L'un ne va pas sans l'autre. Ce cas démontre enfin qu'il s'agit d'un processus de conception spécifique qui dure une décennie et mobilise l'équivalent de cent personnes-années. On mesure ici l'ampleur de la tâche et les raisons des conclusions des rapports NRC qui soulignent la nécessité d'organiser ce processus en termes d'équipe, de processus et de moyens. Ce n'est qu'en travaillant simultanément sur l'instrument, la donnée, les méthodes d'assimilation et la mise en place d'un « écosystème d'usage » (qui existait déjà dans le cas de la $\mathrm{PNT}^{18}$ ) que l'on peut éviter la seconde vallée de la mort. Tout laisse à croire que cette simultanéité jouera un rôle d'autant plus fondamental que la donnée sera innovante et que l' " écosystème d'acteurs » sera à construire.

\section{Bibliographie}

- Abernathy W., Utterback J., 1978. Patterns of industrial innovation. Technology Review, 80, 2-9.

- Andersson E., Hollingworth A., Kelly G., Lönnberg P., Pailleux J., Zhang Z., 1991. Global observing experiment on operational statistical retrievals of satellite sounding data. Mon. Weather Rev., 119, 1851-1864.

- Andersson, E Thépaut J-N., 2008. Ecmwf's 4D-VAR data assimilation system - the genesis and ten years in operations. ECMWF Newsletter, 115, 8-12.

- Bauer P., Thorpe A., Brunet G., 2015. The quiet revolution of numerical weather prediction. Nature, 525, 47-55.

- Cirac G., 2014. Polder and the age of space earth sciences. A study of technological satellite data practices. Thèse de doctorat. Paris: EHESS.

- Conway E., 2008. Atmospheric Science at NASA. A History. Baltimore: The John Hopkins University Press.

- Courtier P., 1997. Variational Methods. J. Meteorol. Soc. Jpn, 75, 211-18.

- Courtier P., Andersson E., Heckley W., Pailleux J., Vasiljevic D., Hamrud M., Hollingworth A., Rabier F., Fisher M.,. 1998. The ECMWF implementation of three-dimensional variational assimilation (3D-VAR). I: Formulation. Q. J. R. Meteorol. Soc., 124, 1783-1807.

- Courtier P., Talagrand O., 1987. Variational assimilation of meteorological observations with the adjoint vorticity equation II : numerical results. Q. J. R. Meteorol. Soc., 113, 13291347.

- _ _ 1990. Variational assimilation of meteorological observations with the direct and adjoint shallow-water equations. Tellus, 42A, 531-549.

- Daget N., 2007. Revue des méthodes d'assimilation. Toulouse: CERFACS.

- Derber J., Wu W-S., 1998. The use of TOVS cloud-cleared radiances in the NCEP SSI analysis system. Mon. Weather Rev., 126, 2287 - 2299.

\footnotetext{
${ }^{17}$ Airs (pour Atmospheric Infrared Sounder) est un sondeur atmosphérique de nouvelle génération lancé par la Nasa sur le satellite Aqua en 2002, dont les performances sont considérablement supérieures à celles à la lignée des sondeurs HIRS (High Resolution Infrared Sounder) lancés à partir de 1979.

${ }^{18}$ Dans le domaine de la PNT, l'écosystème d'usage est existant (les modèles, centres de recherche, centre opérationnel sont déjà là) et très structuré par l'action de l'OMM. D'autres exemples montrent que ce n'est pas toujours le cas (voir par exemple Maniak et al., 2014 pour un aperçu du cas de l'océanographie).
} 
- Errico R., 1997. What is an adjoint model ? Bull. Am. Meteorol. Soc., 78, 2577-2591.

- Eyre J., 2007. Progress achieved on assimilation of satellite data in NWP over the last 30 years, Seminar on Recent Development in the Use of Satellite Observations in NWP. ECMWF, Reading, UK:

- Eyre J., Kelly G., McNally A., Andersson E., Persson A., 1993. Assimilation of TOVS radiance information through one-dimensional variational analysis. Q. J. R. Meteorol. Soc., 119, 1427-1463.

- Geels F., 2005. The dynamics of transitions in socio-technical systems: a multi-level analysis of the transition pathway from horse-drawn carriages to automobiles (1860-1930). Technology Analysis \& Strategic Management, 17, 445-476.

- Henderson R., Clark K., 1990. Architectural Innovation: the reconfiguration of existing product technologies and the failure of established firms. Administrative Science Quarterly, 35, 9-30.

- Hollingsworth A., 1990. Assimilation of remotely sensed atmospheric data from new satellite system in the 1990's, Le Dimet F., Talagrand O., Proceedings of the World Meteorological Organization International Symposium on Assimilation of Observations in Meteorology and Oceanography. Clermont-Ferrand: I-19 - I-25.

- Kalnay E., 2003. Atmospheric Modeling, Data Assimilation and Predictability. Cambridge: Cambridge University Press.

- Le Dimet F-X., Talagrand O., 1986. Variational algorithms for analysis and assimilation of meteorological observations: theoretical aspects. Tellus, 36A, 97-110.

- Lenfle S., 2015. De la valeur des données spatiales : le cas de l'assimilation des radiances en prévision numérique du temps Rapport de recherche. Paris: CNES / CRG - Ecole Polytechnique,

- Mahfouf J-F., Moisselin J-M., Autonès F., Vidot J., 2017. Apport de L'observation satellitaire pour La prévision du temps. La Météorologie, 97 27-33.

- Maniak R., Midler C., Lenfle S., Le Pellec-Dairon M., 2014. Value Management for Exploration Projects. Project Management Journal, 45, 55-66.

- NRC. 2003. Satellite Observations of the Earth's Environment Accelerating the Transition of Research to Operations. Washington DC: National Academy Press.

- Pailleux J., 1996. Impact des mesures satellitaires sur la prévision numérique. La météorologie, 8, 5-18.

- Pailleux J., Geleyn J-F., El-Khatib R., Fischer C., Hamrud M., Thepaut J-N., Rabier F., Andersson E., Salmond D., Burridge D., 2015. Les 25 ans du système de prévision numérique du temps IFS/Arpege. La météorologie, (89),1-10.

- Rabier F., Järvinen H., Klinker E., Mahfouf J-F., Simmons A., 2000. The ECMWF operational implementation of four-Dimensional variational assimilation. I: experimental results with simplified physics. Q. J. R. Meteorol. Soc., 126, 1143-1170.

- Rabier F., Mahfouf J-F., Klinker E., 2000. Une nouvelle technique d'assimilation des données d'observation au CEPMMT: l'assimilation variationnelle Quadridimensionnelle. La météorologie, 8, 87-101.

- Talagrand O., 1997. Assimilation of observations, an introduction. J. Meteorol. Soc. Jpn, 75(1B), 191-209.

_ _ _ 1981a. On the mathematics of data assimilation. Tellus, 33, 321-339.

- $\ldots$. 1981b. A study of the dynamics of four-dimensional data assimilation. Tellus, 33, 4360.

- Talagrand O., Courtier P., 1987. Variational assimilation of meteorological observations with the adjoint vorticity equation. I: theory. Q. J. R. Meteorol. Soc, 113, 1311-1328. 
- Thepaut J-N., Moll P., 1990. Variational inversion of simulated TOVS Radiances using the adjoint technique. Q. J. R. Meteorol. Soc, 116, 1425-1448.

- Thepaut J-N., Mahfouf J-N., 2003. Les potentiels des nouvelles mesures satellitaires pour la prévision numérique. La météorologie, 40, 86-91.

- Tracton M., Desmarais A., VanHaaren R., McPherson R., 1980. The impact of satellite soundings on the national meteorological center's analysis and forecast system - the data system test results. Mon. Weather Rev, 108, 543-586.

- Woods A., 2005. Medium-Range Weather Prediction. The European Approach. The Story of the European Centre for Medium-Range Weather Forecasts. Springer. 